\title{
Energy-Efficient Relaying Strategy with Network Coding in Two-Way Parallel Channels
}

\author{
Ning Hu, ${ }^{1}$ Xiang Chen, ${ }^{1}$ Xiaofeng Zhong, ${ }^{1}$ Ben Slimane, ${ }^{2}$ Yunzhou Li, ${ }^{1}$ and Jing Wang1 \\ ${ }^{1}$ Research Institute of Information Technology, Tsinghua University, Beijing 100084, China \\ ${ }^{2}$ Communication Systems, Royal Institute of Technology (KTH), 16440 Stockholm, Sweden
}

Correspondence should be addressed to Xiang Chen, chenxiang98@mails.tsinghua.edu.cn

Received 25 November 2010; Accepted 11 January 2011

Academic Editor: A. Maaref

Copyright (c) 2011 Ning Hu et al. This is an open access article distributed under the Creative Commons Attribution License, which permits unrestricted use, distribution, and reproduction in any medium, provided the original work is properly cited.

\begin{abstract}
We consider a two-way communication assisted by parallel regenerative decode-and-forward relays operating in orthogonal channels. In a system with limited channel state information at each source and relay node, an optimum distributed power allocation strategy is proposed to minimize the total transmit power, providing a target signal-to-noise ratio at each destination with a target outage probability. Moreover, combined with opportunistic relaying and network coding, a distributed decision mechanism is proposed for the relay node to decide whether to help the transmission or not. In this proposal, each source works out the transmit power and the decision threshold then broadcasts them. The selected relay compares the decision threshold with the channel gain of its weaker relay-to-destination link, then determines whether to forward the networkcoded data or not. Simulation results show the advantage of this strategy in terms of energy efficiency for a two-hop two-way communication scenario. The proposed strategy is very flexible as it can trade outage to power consumption and vice versa.
\end{abstract}

\section{Introduction}

Wireless relaying offers space diversity to extend the transmission range and to enhance end-to-end transmission performance such as outage probability and data rate. In conventional single link communication, the transmission between the source and the destination suffers from severe fading due to multipath fading effects and path loss, which results in unreliable communication. Fortunately, relays can provide cooperative diversity [1], and save power to improve the reliability without the need of physical antenna arrays. Recently, some works such as [2-4] introduced network coding [5] to the bidirectional cooperation. Especially, [2, 3] focus on the bit-level transmission, and working out the optimum system throughput provided that both the two source-to-relay channels are better than the direct channel between the two sources in the three-node model. As shown in Figure 1, two source nodes $\left(S_{1}\right.$ and $\left.S_{2}\right)$ exchange messages with the help of a relay (namely, $R$ ), using time-division scheme in two-way communications. The traditional method [2] needs four phases (Figure 1(a)) to complete the exchange of information. However, by using network coding (a bitwise XOR operation at the relay node), only three phases are needed (Figure 1(b)). In such a three-phase relaying scheme, the messages from $S_{1}$ and $S_{2}$ are first decoded at the relay node, network coded, re-encoded, and then sent as one message to both destinations simultaneously. Here in this paper, we extend the three-node case to the scenario in which there are multiple relay candidates that could assist the source nodes in the two-way communication as shown in Figure 2.

When there are multiple relay node candidates, the multiple relays could simultaneously assist the transmission [6-8], or the most suitable single relay could be selected for transmission according to the channel state information (CSI). This opportunistic idea is based on instantaneously selecting an "on-peak" receiver with the "good" channel condition to improve system performance [9-12]. These works focused on the multirelay transmission in one-way transmissions (i.e., the messages are sent from $S_{1}$ to $S_{2}$, but no messages come from $S_{2}$ to $S_{1}$ ). Meanwhile, they 
assume that either the destination cannot directly receive the signals from the source [9], or that the complete CSI of all communication channels can be available at the source node $[10,12]$.

In addition, from the control mechanism's point of view, the cooperative strategies can be categorized into two types: central control strategy and distributed strategy. In most cases of practical systems, the distributed strategy is more practical since it only needs local and partial CSI, which overcomes the obstacles of a centralized architecture such as the substantial feedback requirements, overhead and delay, and so forth. For example, in [13], users select cooperation partners based on a priority list in a distributed manner for multiuser networks with coded cooperation. Reference [14] provides a relay selection algorithm based on instantaneous channel measurements obtained by each relay node locally, which reduces the cooperative costs among relays work in but only the one-way transmission is considered in $[8,13$, $14]$.

Especially in [8], several optimum distributed power allocation strategies minimize the total transmit power while providing a target signal-to-noise ratio at the destination with a target outage probability. Due to the one-way transmission, [8] only needs to consider the relay-to-destination link after the relays successfully decode the messages. When network coding is introduced to decrease the transmission phases in two-way communications, as shown in Figure 1(b), the outage probability should be investigated on both $R \leftrightarrow S_{1}$ and $R \leftrightarrow S_{2}$ links in the third transmission phase after the relay has correctly decoded the messages from the two source nodes.

In this paper, our design target is to maximize power efficiency in two-way relay systems. Due to the limited knowledge of CSI at both source nodes, our distributed strategy mainly focuses on the forwarding decision and distributed power allocation. Firstly, a single relay node will be chosen as the opportunistic relay. Then, the opportunistic relay makes a decision whether to forward the source data according to a proper forwarding threshold. Moreover, the distributed power allocation based on the decode-andforward (DaF) scheme [15] works out the corresponding transmit powers $\left(P_{S_{1}}, P_{S_{2}}\right)$ and the forwarding threshold $\left(\alpha_{t}\right)$ at the source nodes. The source nodes broadcast the optimum pair $\left(\left(P_{S_{1}}, \alpha_{t}\right)\right.$ and $\left.\left(P_{S_{2}}, \alpha_{t}\right)\right)$ to be used by the relay nodes.

The remainder of this paper is organized as follows. The next section describes the system model. The analysis of the distributed strategy is performed in Section 3. Simulation results can be found in Section 4. Finally, concluding remarks are given in Section 5.

\section{System Model and Background}

As shown in Figure 2, we consider a relay network consisting of an $S_{1} \leftrightarrow S_{2}$ two-way pair and $M$ relay nodes employing DaF. For simplicity, we denote relay $i$ by $R_{i}$. We assume that the relaying operates in orthogonal time-division channels. Let $h_{1 i}$ and $h_{2 i}$ denote the fading coefficient of the $S_{1} \leftrightarrow R_{i}$ and $R_{i} \leftrightarrow S_{2}$ channels for the $i$ th relay node, respectively, $i=1, \ldots, M$. The fading coefficient of the $S_{1} \leftrightarrow S_{2}$ link is denoted by $h_{0}$. Assume that each channel is slowly varying flat fading, and $\left\{h_{1 i}, h_{2 i}, h_{0}\right\}$ are all independent realizations of zero-mean complex Gaussian random variables with variance $\sigma_{1 i}^{2}, \sigma_{2 i}^{2}$ and $\sigma_{0}^{2}$, respectively. We define $\beta_{1 i}=1 / \sigma_{1 i}^{2}$, $\beta_{2 i}=1 / \sigma_{2 i}^{2}$, and $\beta_{0}=1 / \sigma_{0}^{2}$.

Firstly, the relay selection is prior to the forwarding decision and the distributed power allocation. That is, in the opportunistic relaying, the "good" relay is chosen prior to the transmission among a collection of $M$ possible candidates and source nodes. As shown in Figure 1(b), in the first transmission phase, $S_{1}$ broadcasts $X_{1}$ with power $P_{S_{1}}$, then $S_{2}$ broadcasts $X_{2}$ with power $P_{S_{2}}$. The corresponding destinations (i.e., $S_{2}$ node and $S_{1}$ node) observe $y_{2}$ and $y_{1}$ as

$$
\begin{aligned}
& y_{2}=\sqrt{P_{S_{1}}} h_{0} X_{1}+Z_{2}, \\
& y_{1}=\sqrt{P_{S_{2}}} h_{0} X_{2}+Z_{1},
\end{aligned}
$$

and the opportunistic relay $R_{r}$ observes $y_{1 r}$ from the $S_{1} \leftrightarrow R_{r}$ link and $y_{2 r}$ from the $S_{2} \leftrightarrow R_{r}$ as

$$
\begin{aligned}
& y_{1 r}=\sqrt{P_{S_{1}}} h_{1 r} X_{1}+Z_{1 r}, \\
& y_{2 r}=\sqrt{P_{S_{2}}} h_{2 r} X_{2}+Z_{2 r},
\end{aligned}
$$

where $Z_{2}, Z_{1}, Z_{1 r}$, and $Z_{2 r}$ are additive white Gaussian noise (AWGN) terms at the corresponding destinations and the selected relay, respectively. Without loss of generality, they are of variance $N_{0}$.

The opportunistic relay is chosen based on the following criterion:

$$
\begin{gathered}
h_{i}=\min \left\{\left|h_{1 i}\right|^{2},\left|h_{2 i}\right|^{2}\right\}, \quad i=1, \ldots, M, \\
r=\underset{i}{\operatorname{argmax}}\left\{h_{i}\right\},
\end{gathered}
$$

which is similar to that in $[9,16]$.

That is, a single "good" relay is selected based on the end-to-end instantaneous wireless channel conditions from the $M$ relay candidates to act as the cooperative partner. Among the $M$ relay candidates, the relay node that maximizes $h_{i}$ is defined as the "good" relay $R_{r}$. We denote $\min \left\{\left|h_{1 r}\right|^{2},\left|h_{2 r}\right|^{2}\right\}$ by $A_{r}$. We use this relay selection criterion because the quality of the signals received by each destination depends on the quality of the weaker link [16]. Assume this opportunistic relay node can decode $X_{1}$ and $X_{2}$ correctly when its received signal-to-noise ratio (SNR) from $S_{1}$ and $S_{2}$ satisfies

$$
\begin{aligned}
& \Gamma_{1 r}=\frac{P_{S_{1}}\left|h_{1 r}\right|^{2}}{N_{0}} \geq \gamma_{\text {target }}, \\
& \Gamma_{2 r}=\frac{P_{S_{2}}\left|h_{2 r}\right|^{2}}{N_{0}} \geq \gamma_{\text {target }},
\end{aligned}
$$




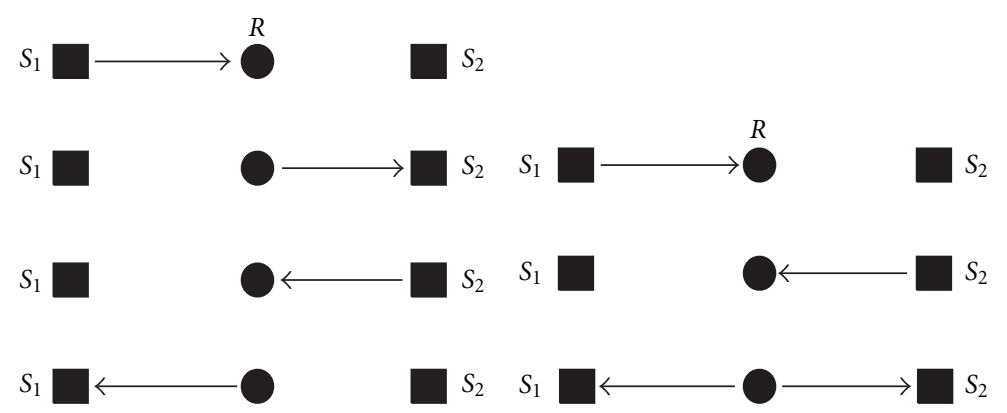

(a)

(b)

Figure 1: (a) Traditional relaying; (b) three-phase relaying.

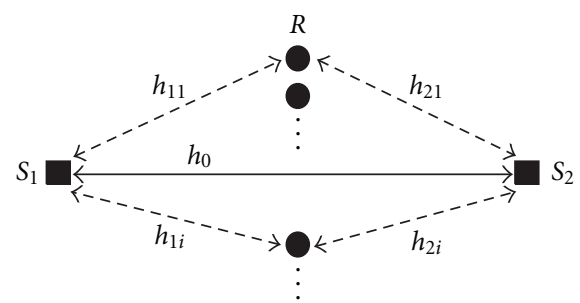

Figure 2: Two-way communication with multiple candidates.

where $\gamma_{\text {target }}$ is the given SNR constraint for correctly decoding. By using network coding, a bitwise XOR operation at the selected relay node encodes the messages from both $S_{1}$ and $S_{2}$, then the encoded messages are sent to each destination node where $S_{1}$ and $S_{2}$ could get the desired messages by performing an XOR operation [5]. Thus, it only needs three phases (Figure 1(b)). The destination processes, the messages from the source, and the relay jointly when the decoder operates with log likelihood ratios [2] to achieve the performance gain of maximal ratio combining (MRC). Then, the received SNR at the destination $S_{2}$ is

$$
\Gamma_{2}=\frac{P_{S_{1}}\left|h_{0}\right|^{2}+P_{r}\left|h_{2 r}\right|^{2}}{N_{0}} .
$$

And the resulting SNR at the destination $S_{1}$ is

$$
\Gamma_{1}=\frac{P_{S_{2}}\left|h_{0}\right|^{2}+P_{r}\left|h_{1 r}\right|^{2}}{N_{0}} .
$$

Assume each destination can correctly receive the source data whenever $\Gamma_{2} \geq \gamma_{\text {target }}$ and $\Gamma_{1} \geq \gamma_{\text {target }}$. Based on the assumption above, the problem of power efficiency for $\mathrm{DaF}$ relay networks with parallel channels can be modeled as in Problem $\left(Q_{1}\right)$ :

$$
\begin{array}{ll}
\min _{\left\{\mathrm{P}_{\mathrm{S}_{1}}, \mathrm{P}_{\mathrm{S}_{2}}, \mathrm{P}_{\mathrm{r}}, \gamma\right\}} & \left\{P_{S_{1}}+P_{S_{2}}+P_{r}\right\} \\
\text { subject to } & \frac{P_{S_{1}}\left|h_{0}\right|^{2}+P_{r}\left|h_{2 r}\right|^{2}}{N_{0}} \geq \gamma_{\text {target }} \\
& \frac{P_{S_{2}}\left|h_{0}\right|^{2}+P_{r}\left|h_{1 r}\right|^{2}}{N_{0}} \geq \gamma_{\text {target }} \\
& \frac{P_{S_{1}}\left|h_{1 r}\right|^{2}}{N_{0}} \geq \gamma_{\text {target }} \\
& \frac{P_{S_{2}}\left|h_{2 r}\right|^{2}}{N_{0}} \geq \gamma_{\text {target. }} .
\end{array}
$$

Since the traditional two-way relaying is decomposed into two one-way transmissions (Figure 1(a)), the optimum power allocation strategy for one-way transmission in DaF relay networks [8] is briefly restated as follows. Assume the one-way transmission is from $S_{1}$ to $S_{2}$, then [8] works out the optimum power $P_{S_{1}}^{*}$ and the forwarding threshold $\alpha_{t} \cdot P_{S_{1}}^{*}$ can only be one of $M+1$ discrete values $\left\{\gamma_{\text {target }} N_{0} /\left|h_{0}\right|^{2}, \gamma_{\text {target }} N_{0} /\left|h_{11}\right|^{2}\right.$, $\left.\gamma_{\text {target }} N_{0} /\left|h_{12}\right|^{2}, \ldots, \gamma_{\text {target }} N_{0} /\left|h_{1 M}\right|^{2}\right\}$. The parameters $\left\{P_{S_{1}}^{*}, \gamma\right\}$ are obtained based on that the channel gain of relayto-destination, $\left|h_{2 i}\right|^{2}$ is exponentially distributed, which does not find out the "bottle-neck" of transmission (i.e., the weaker link of the relays). When relaying is selected (i.e., $P_{S_{1}}^{*}<\gamma_{\text {target }} N_{0} /\left|h_{0}\right|^{2}$ ), the source node broadcasts $\left\{P_{S_{1}}^{*}, \alpha_{t}\right\}$ to all the candidates. If the channel gain of relaydestination link is greater than the forwarding threshold, namely, $\left|h_{2 i}\right|^{2} \geq \alpha_{t}$, then the selected relays forward the source data. It implies that multiple relays could participate in the relaying simultaneously.

In the model with network coding and opportunistic relaying, as shown in Figure 1(b), when relaying is selected, $P_{S_{1}}^{*}=\gamma_{\text {target }} N_{0} /\left|h_{1 r}\right|^{2}$, the target SNR can be guaranteed at the selected relay during the transmission from $S_{1}$ to the selected relay. And it is the same to $S_{2}$ when $P_{S_{2}}^{*}=$ $\gamma_{\text {target }} N_{0} /\left|h_{2 r}\right|^{2}$. But the outage event may occur in the third 
transmission phase because the instantaneous channel gain of forwarding links may be less than $\alpha_{t}$, which is calculated by the statistics of all the links. Thus, given the target SNR, $\gamma_{\text {target}}$, at each destination with a target outage probability, $\rho_{\text {target }}$, in two-way communications, we rewrite Problem $\left(Q_{1}\right)$ as Problem $\left(Q_{2}\right) . E\left[P_{r}\right]$ is the expected value of the transmit power of opportunistic relay. Problem $\left(Q_{2}\right)$ is the problem of power efficiency in two-way relay networks.

$$
\begin{array}{ll}
\min _{\left\{\mathrm{P}_{\mathrm{S}_{1}}, \mathrm{P}_{\mathrm{S}_{2}}, \mathrm{P}_{\mathrm{r}}, \gamma\right\}} & \left\{P_{S_{1}}+P_{S_{2}}+E\left[P_{r}\right]\right\} \\
\text { subject to } & P_{\text {rob }}\left\{\frac{P_{S_{1}}\left|h_{0}\right|^{2}+P_{r}\left|h_{2 r}\right|^{2}}{N_{0}} \leq \gamma_{\text {target }}\right\} \leq \rho_{\text {target }} \\
& P_{\text {rob }}\left\{\frac{P_{S_{2}}\left|h_{0}\right|^{2}+P_{r}\left|h_{1 r}\right|^{2}}{N_{0}} \leq \gamma_{\text {target }}\right\} \leq \rho_{\text {target }} \\
& \frac{P_{S_{1}}\left|h_{1 r}\right|^{2}}{N_{0}} \geq \gamma_{\text {target }} \\
& \frac{P_{S_{2}}\left|h_{2 r}\right|^{2}}{N_{0}} \geq \gamma_{\text {target }} .
\end{array}
$$

Obviously, the transmission through the relaying could be more power efficient than the direct transmission on the condition that each relaying link is better than the direct link (i.e., $\left|h_{1 r}\right|^{2} \geq\left|h_{0}\right|^{2}$ and $\left|h_{2 r}\right|^{2} \geq\left|h_{0}\right|^{2}$ ). In this paper, we will work out this problem.

\section{Distributed Relaying Decision and Power Allocation Strategy}

In this section, a distributed relaying decision mechanism followed by distributed power allocation strategy is proposed to crack the obstacles of centralized mechanism, using the limited CSI at each node.

When $S_{1}$ transmits the training messages, due to the broadcast nature of wireless medium, all relay nodes and $S_{2}$ can simultaneously estimate their $S_{1} \leftrightarrow R$ and $S_{1} \leftrightarrow$ $S_{2}$ fading coefficients $\left\{h_{1 i}, i=1, \ldots, M\right\},\left\{h_{0}\right\}$, respectively. Similarly, when the relay and $S_{2}$ transmit the training bits, $\left\{h_{0}, h_{11}, h_{12}, \ldots, h_{i M}\right\}$ can be estimated at $S_{1}$. However, $\left\{h_{21}, h_{22}, \ldots, h_{2 M}\right\}$ may not be available at $S_{1}$. Thus, the distributed strategy is proposed with the realizations $\left\{h_{0}, h_{1 i}, h_{12}, \ldots, h_{1 M}\right\}$, the statistics of all the links available at the source $S_{1}$, and the realizations $\left\{h_{0}, h_{21}, h_{22}, \ldots, h_{2 M}\right\}$, the statistics of all the links available at $S_{2}$. The relay nodes are assumed to have their local CSI, that is, $h_{1 i}$ and $h_{2 i}$ for $R_{i}, i=1,2, \ldots, M$.

The nature of the distributed strategy requires that each relay should make its decision only on its local CSI. Since the opportunistic relay has been chosen in advance (as mentioned in Section 2), the selected relay only needs to decide whether to forward the source data or not. In this paper, the selected relay broadcasts the network-coded data when its minimal channel gain of relay-to-destination satisfies

$$
A_{r}=\min \left\{\left|h_{1 r}\right|^{2},\left|h_{2 r}\right|^{2}\right\} \geq \alpha_{t},
$$

where $\alpha_{t}$ is the forwarding threshold value. One reason is because this network coding needs both ends to decode at the same rate, another reason is that the transmission data rate broadcast by the relay is limited by the weaker link. Thus, the opportunistic relay forwards the decoded signals with sufficient power $P_{r}^{*}$

$$
\begin{aligned}
P_{r}^{*} & =\frac{\gamma_{\text {target }}^{\prime} N_{0}}{A_{r}}=\frac{\max \left\{0, \gamma_{\text {target }} N_{0}-P_{S k}^{*}\left|h_{k r}\right|^{2}\right\}}{\min \left\{\left|h_{1 r}\right|^{2},\left|h_{2 r}\right|^{2}\right\}} \\
& \leq \frac{\max \left\{0, \gamma_{\text {target }} N_{0}-P_{S k}^{*}\left|h_{k r}\right|^{2}\right\}}{\alpha_{t}},
\end{aligned}
$$

where $\gamma_{\text {target }}^{\prime}$ denotes the SNR contribution from the relay.

We note that such a distributed decision mechanism results in a nonzero probability that none of the relay nodes satisfies (7), and hence a nonzero outage probability. A large value of $\alpha_{t}$ means that the selected relay will transmit with less power and less often, which saves power but at the expense of a high outage probability. On the other hand, a small value for $\alpha_{t}$ means that the selected relay will transmit possibly higher power and more often, which results in a low outage probability but possibly high power. Hence, the source nodes should work out the pairs $\left\{P_{S_{1}}^{*}, \alpha_{t}\right\}$ and $\left\{P_{S_{2}}^{*}, \alpha_{t}\right\}$ to meet a system-given specification, that is, an outage probability requirement $\rho_{\text {target }}$.

From the source's point of view, the transmit power of the selected relay node is a random variable with the known statistics, since the realizations of the forwarding link are not available at the source node. Due to (3), we have the cumulative distribution function (CDF) of $A_{r}$

$$
F_{A_{r}}(x)=\prod_{i=1}^{M}\left(1-e^{-\beta_{i} x}\right), \quad x \geq 0,
$$

and its probability density function ( $\mathrm{pdf}$ ) is given by

$$
f_{A_{r}}(x)=\sum_{k=1}^{M} \beta_{k} e^{-\beta_{k} x} \prod_{i=1, i \neq k}^{M}\left(1-e^{-\beta_{i} x}\right), \quad x \geq 0,
$$

where $\beta_{i}=\sum_{k=1}^{2} \beta_{i k}, i=1, \ldots, M$ (refer to the appendix).

Having the pdf of $A_{r}$, the expected value of the transmit power of the selected relay $R_{r}$ is obtained as

$$
E\left[P_{r}\right]=\int_{\alpha_{t}}^{\infty} \frac{\max \left\{0, \gamma_{\text {target }} N_{0}-P_{S k}\left|h_{0}\right|^{2}\right\}}{x} f_{A_{r}}(x) d x,
$$

where $k=\operatorname{argmax}_{\{j\}}\left\{\left|h_{j r}\right|^{2}\right\}, j=1,2$. The factor $\max \left\{0, \gamma_{\text {target }} N_{0}-P_{S k}\left|h_{0}\right|^{2}\right\}$ means that the relay should pay more power according to the channel gain of weaker link to guarantee the correct decoding at both ends in the third transmission phase. 
From (3) and (7), the forwarding threshold $\alpha_{t}$ should be chosen as the value that satisfies the outage probability with equality, that is,

$$
\rho_{\text {target }}=\operatorname{Pr}\left\{A_{r}<\alpha_{t}\right\}=F_{A_{r}}\left(\alpha_{t}\right)=\prod_{i=1}^{M}\left(1-e^{-\beta_{i} \alpha_{t}^{*}}\right) .
$$

Exact expression for the threshold $\alpha_{t}$ is difficult to obtain but it is possible to derive bounds for it. For instance, by letting $\beta_{\min }=\min \left\{\beta_{1}, \beta_{2}, \ldots, \beta_{M}\right\}$ and $\beta_{\max }=\max \left\{\beta_{1}, \beta_{2}, \ldots, \beta_{M}\right\}$, we can bound the outage probability as

$$
\left(1-e^{-\beta_{\max } \alpha_{t}^{*}}\right)^{M} \leq \prod_{i=1}^{M}\left(1-e^{-\beta_{i} \alpha_{t}^{*}}\right) \leq\left(1-e^{-\beta_{\min } \alpha_{t}^{*}}\right)^{M} .
$$

Therefore, $\alpha_{t}^{*}$ is bounded as

$$
-\frac{1}{\beta_{\max }} \ln \left(1-\rho_{\text {target }}^{1 / M}\right) \leq \alpha_{t}^{*} \leq-\frac{1}{\beta_{\min }} \ln \left(1-\rho_{\text {target }}^{1 / M}\right) .
$$

The value of $\alpha_{t}^{*}$ can be obtained by a search in the given range in (15) numerically.

In addition, the transmit power of the source nodes can be calculated by Theorem 1, which provides the following optimal solution.

Theorem 1. The optimum transmit power of the source nodes, $P_{S_{1}}^{*}$ and $P_{S_{2}}^{*}$, can only be one of the discrete values in the following sets:

$$
\begin{aligned}
& \left\{\frac{\gamma_{\text {target }} N_{0}}{\left|h_{1 r}\right|^{2}}, \frac{\gamma_{\text {target }} N_{0}}{\left|h_{0}\right|^{2}}\right\}, \\
& \left\{\frac{\gamma_{\text {target }} N_{0}}{\left|h_{2 r}\right|^{2}}, \frac{\gamma_{\text {target }} N_{0}}{\left|h_{0}\right|^{2}}\right\} .
\end{aligned}
$$

respectively.

Each source node's transmit power is equal to $\gamma_{\text {target }} N_{0} /\left|h_{0}\right|^{2}$ when the direct transmission is preferred. And the source nodes transmit power $\gamma_{\text {target }} N_{0} /\left|h_{1 r}\right|^{2}, \gamma_{\text {target }} N_{0} /\left|h_{2 r}\right|^{2}$, respectively, when the relaying is preferred. Thus, the power consumption of relaying is less than that of direct transmission, that is,

$$
\begin{aligned}
& P_{S_{1}}^{*}+\int_{\alpha_{t}^{*}}^{\infty} \frac{\max \left\{0, \gamma_{\text {target }} N_{0}-P_{S_{1}}^{*}\left|h_{0}\right|^{2}\right\}}{x} f_{A_{r}}(x) d x \leq \frac{\gamma_{\text {target }} N_{0}}{\left|h_{0}\right|^{2}}, \\
& P_{S_{2}}^{*}+\int_{\alpha_{t}^{*}}^{\infty} \frac{\max \left\{0, \gamma_{\text {target }} N_{0}-P_{S_{2}}^{*}\left|h_{0}\right|^{2}\right\}}{x} f_{A_{r}}(x) d x \leq \frac{\gamma_{\text {target }} N_{0}}{\left|h_{0}\right|^{2}} .
\end{aligned}
$$

Proof. Here, the distributed strategy is based on the opportunistic relaying and network coding. Due to the opportunistic relaying, the "good" relay is chosen prior to the transmission (3). And the source nodes can work out the forwarding threshold $\alpha_{t}$ with the known statistics of all the links ( $\alpha_{t}$ is the same to both $S_{1}$ node and $S_{2}$ node) by (15). Then, $S_{1}$ node can evaluate (18) to make a decision whether to perform relaying based on the knowledge of $h_{0}$ and $h_{1 r}$ (for $P_{S_{1}}^{*}$ ). At the same time, $S_{2}$ node can also evaluate (19) based on the knowledge of $h_{0}$ and $h_{2 r}$ (for $P_{S_{2}}^{*}$ ). Consequently, $S_{1}$ and $S_{2}$ nodes can exchange "1 bit" signaling information (here, this " 1 bit" signaling information is little overhead and can be achieved by a one-time handshaking protocol between the two source nodes.) to finally decide whether to transmit the source data by the direct link or relaying link.

We consider the scenario in which the relaying is preferred. Clearly, the target SNR at the selected relay can be achieved without an outage event in each source-to-relay link with the source transmit power $\gamma_{\text {target }} /\left|h_{1 r}\right|^{2}, \gamma_{\text {target }} /\left|h_{2 r}\right|^{2}$, respectively.

Since each source node only knows the statistics of all the links without the knowledge of the realizations of channel gain in the relay-to-destination link, the outage event may occur in the third phase. Due to (13), the target outage probability can be satisfied when the forwarding threshold $\alpha_{t}^{*}$ is calculated from (15). In the following content, it can be shown that the target SNR at destination can be satisfied. Assume the $R_{r} \leftrightarrow S_{2}$ link is weaker than the $R_{r} \leftrightarrow S_{1}$ link, that is, $\left|h_{2 r}\right|^{2} \leq\left|h_{1 r}\right|^{2}$. From (8), we can get $P_{r}^{*}=\left(\max \left\{0, \gamma_{\text {target }} N_{0}-P_{S 1}^{*}\left|h_{0}\right|^{2}\right\}\right) /\left|h_{2 r}\right|^{2}$, $P_{S_{1}}^{*}=\gamma_{\text {target }} N_{0} /\left|h_{1 r}\right|^{2} \leq \gamma_{\text {target }} N_{0} /\left|h_{2 r}\right|^{2}$ (i.e., the transmit power of $S_{1}$ is less than that of souce $\left.2, P_{S_{1}}^{*} \leq P_{S_{2}}^{*}\right)$.

At $S_{2}$, the received SNR is

$$
\begin{aligned}
& P_{S_{1}}^{*}\left|h_{0}\right|^{2}+P_{r}^{*}\left|h_{2 r}\right|^{2} \\
& \quad=P_{S 1}^{*}\left|h_{0}\right|^{2}+\frac{\max \left\{0, \gamma_{\text {target }} N_{0}-P_{S_{1}}^{*}\left|h_{0}\right|^{2}\right\}}{\left|h_{2 r}\right|^{2}}\left|h_{2 r}\right|^{2} \\
& \quad \geq \gamma_{\text {target }} .
\end{aligned}
$$

At $S_{1}$, the received SNR is

$$
\begin{aligned}
& P_{S_{2}}^{*}\left|h_{0}\right|^{2}+P_{r}^{*}\left|h_{1 r}\right|^{2} \\
& \quad=P_{S_{2}}^{*}\left|h_{0}\right|^{2}+\frac{\max \left\{0, \gamma_{\text {target }} N_{0}-P_{S_{1}}^{*}\left|h_{0}\right|^{2}\right\}}{\left|h_{2 r}\right|^{2}}\left|h_{1 r}\right|^{2} \\
& \quad \geq P_{S_{2}}^{*}\left|h_{0}\right|^{2}+\frac{\max \left\{0, \gamma_{\text {target }} N_{0}-P_{S_{1}}^{*}\left|h_{0}\right|^{2}\right\}}{\left|h_{2 r}\right|^{2}}\left|h_{2 r}\right|^{2} \\
& \geq \gamma_{\text {target }} .
\end{aligned}
$$

Using the expression in (12), the expected value of total transmit power is as follows:

$$
\begin{aligned}
E\left[P_{\text {total }}\right]= & P_{S_{1}}+P_{S_{2}}+E\left[P_{r}\right] \\
= & P_{S_{1}}+P_{S_{2}} \\
& +\int_{\alpha_{t}^{*}}^{\infty} \frac{\max \left\{0, \gamma_{\text {target }} N_{0}-P_{S_{1}}\left|h_{0}\right|^{2}\right\}}{x} f_{A_{r}}(x) d x .
\end{aligned}
$$

Therefore, the target SNR and the target outage probability can be satisfied. Theorem 1 is proved. 
TABLE 1: Energy-efficient relaying strategy with network coding in two-way parallel channels.

Prior to the transmission in two-way relaying:

opportunistic relay selection according to (3).

Start the transmission:

at $S_{1}$ node, $S_{2}$ node:

(1) decide whether to transmit source data by the direct link or by the relaying link according to (18) and (19);

(2) if both (18) and (19) are satisfied, the forwarding threshold is calculated by formula (15), the transmit power of each source node is calculated by (16) and (17);

Otherwise the direct transmission is preferred, the transmit power of each source node is computed by (16) and (17);

(3) the source nodes start the transmission including broadcasting the message of transmit power and $\alpha_{t}$.

At the selected relay node (if the relaying is preferred):

(1) decide whether to broadcast the network-coded data by (7);

(2) work out the transmit power of relay node by (8).

The proposed "energy-efficient relaying strategy with network coding" in two-way parallel channels is summarized in Table 1. Here, the opportunistic relaying is applied to decrease the cooperative costs, to save power, and capture the nice link for the practical system. Moreover, the network coding is introduced to save power.

\section{Simulation Results}

In this section, we will consider the following three different cooperative schemes.

(i) The proposed distributed strategy (for relaying decision and power allocation) combined with opportunistic relaying and network coding (DSON for short).

(ii) The optimum distributed power allocation (ODPA) scheme [8].

(iii) Single relay model (SRM) [8].

The traditional two-way relaying is decomposed into two one-way transmissions (as shown in Figure 1(a)). Moreover, to the best of our knowledge, (ii) and (iii) outperform the existing distributed schemes in one-way relaying, so we focus on comparing the performance of (i) with that of (ii) and (iii). We consider the two-way transmission consisting of $S_{1}$ node and $S_{2}$ node $100 \mathrm{~m}$ apart, and $M$ relay nodes between the source nodes. The fading model is considered as [15], that is, the variance of the channel gain is proportional to the distance between nodes. Here, we have

$$
\begin{gathered}
\sigma_{k i}^{2}=\frac{0.5 C}{d_{S_{k} R_{i}}^{\alpha}}, \quad k=1,2, \\
\sigma_{0}^{2}=\frac{0.5 C}{d_{S_{1} S_{2}}^{\alpha}},
\end{gathered}
$$

where $d_{j i}$ is the distance between node $j$ and node $i$, the path-loss exponent is denoted by $\alpha$, the factor 0.5 is due to

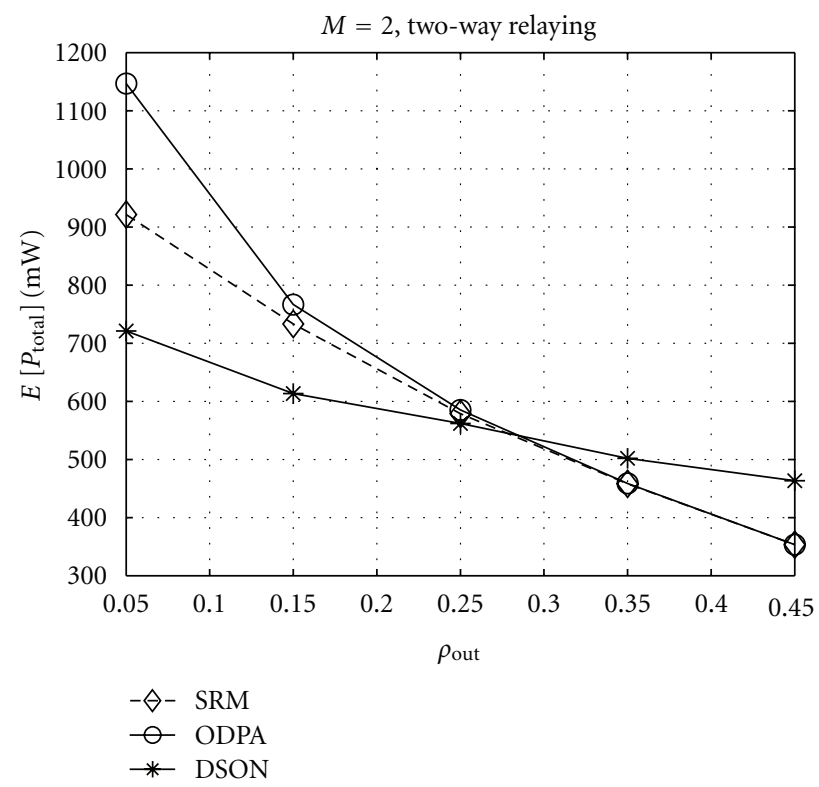

FIGURE 3: $E\left[P_{\text {total }}\right]$ versus the outage probability $\rho_{\text {out }}$ for the different distributed strategies with $\left\{d_{S_{1} R_{i}}\right\}_{i=1}^{M}=\{0.2,0.8\},\left\{d_{R_{i} S_{2}}\right\}_{i=1}^{M}=$ $\{0.8,0.2\}$, and $M=2$.

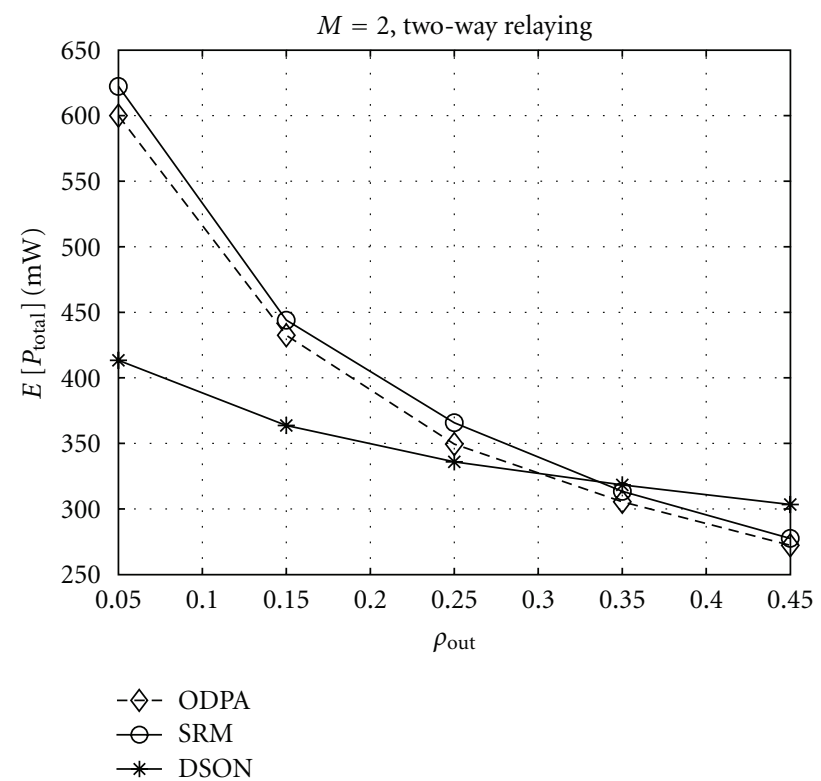

FIGURE 4: $E\left[P_{\text {total }}\right]$ versus the outage probability $\rho_{\text {out }}$ for the different distributed strategies with $M=2$.

the above variances defined by two dimension. $C$ is a constant that is expressed as $C=G_{t} G_{r} \lambda^{2} / L(4 \pi)^{2}$, where $G_{t}$ is the transmitter antenna gain, $G_{r}$ is the receiver antenna gain, $\lambda$ is the wavelength, and $L$ is the system loss factor not related to propagation $(L \geq 1)$. The values $\alpha=3, G_{t}=G_{r}=1$, $\lambda=(1 / 3) m, L=1$, are used in the simulations. Assume the AWGN variances on all links to be $N_{0}=10^{-10}$ and the target $\operatorname{SNR}\left(\gamma_{\text {target }}\right)$ to be $10 \mathrm{~dB}$. 


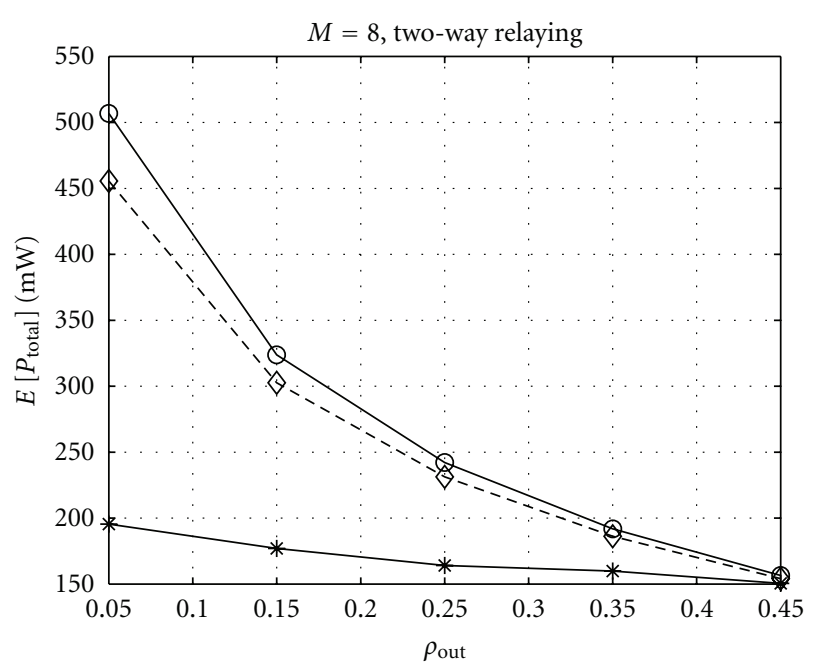

$-\diamond-$ ODPA
$\ominus-$ SRM
$*$ DSON

FIGURE 5: $E\left[P_{\text {total }}\right]$ versus the outage probability $\rho_{\text {out }}$ for the different distributed strategies with $M=8$.

We first consider the case in which $\left\{d_{S_{1} R_{i}}\right\}_{i=1}^{M}=\{0.2,0.8\}$, $\left\{d_{R_{i} S_{2}}\right\}_{i=1}^{M}=\{0.8,0.2\}$, and $M=2$. Figure 3 illustrates the numerical results of the expected value of the total power $E\left[P_{\text {total }}\right]$ as a function of the target outage probability $\rho_{\text {out }}$. It is observed that the proposed strategy (DSON) outperforms the existing schemes at practical values of outage probability. For instance, for an outage $\rho_{\text {out }}=0.05$, approximately $22 \%$, $37 \%$ is saved by the DSON scheme as compared to the ODPA and the SRM schemes, respectively. The performance of the DSON scheme is not better than that of the ODPA and the SRM at the higher outage probability regimes but we know, as fact, that the high outage probability is prohibited in practical systems.

Generally, the relays are located in the midst of two source nodes. The following simulation results are based on the scenario in which all the relay nodes are in the midst of two source nodes. Namely, $\left\{d_{S_{1} R_{i}}\right\}_{i=1}^{M}=\left\{d_{R_{i} S_{2}}\right\}_{i=1}^{M}=$ $\{0.5, \ldots, 0.5\}$.

Figure 4 illustrates the numerical results of the expected value of the total power $E\left[P_{\text {total }}\right]$ as a function of the target outage probability $\rho_{\text {out }}$ for the case $M=2$. Similarly to the results of Figure 3, Figure 4 shows that the proposed scheme (DSON) outperforms both (ii) and (iii) at the lower $\rho_{\text {out }}$ regime. It is also observed that the relative gain of the proposed scheme is larger in comparison to the case of Figure 3. For instance, at outage of $\rho_{\text {out }}=0.05$, approximately $30 \%, 31 \%$ is saved by the DSON with respect to the ODPA and the SRM, respectively. It is clear that, when the relays are in the middle between the two source nodes, they provide good help to both source nodes. With only two relays $(M=2)$, the opportunistic relaying has less spatial channels to choose from (less spacial diversity) and suffers from broadcasting the network-coded data according to the weaker link in the third transmission phase; this is the implication on the performance of the DSON scheme at higher outage probability regime.

Figure 5 illustrates the numerical results for the case when the number of relay nodes is $M=8$. Here, it is observed that the DSON strategy has improved considerably in comparison to the case of one- and two-relay nodes. The DSON strategy now enjoys more spatial diversity in the presence of more relay nodes as the candidates. Though the ODPA and the SRM also have more spatial diversities, both the ODPA and the SRM select relays mainly based on source-to-relay links, so their spatial dimension is half of that of the DSON strategy. Moreover, the DSON with network coding only has three transmission phases, this results in more saving in power consumption.

\section{Conclusions}

In this paper, an energy-efficient relaying strategy with network coding in two-way parallel channels is proposed. In the first stage of this proposal, based on the opportunistic relaying strategy, only one relay is selected to assist the transmission. It decreases the cooperative costs among relays, which is preferred in practical systems. Meanwhile, it guarantees that the DSON chooses the relay from both sourceto-relay and relay-to-destination links. Moreover, network coding decreases the transmission phases from four to three. Combined with the opportunistic relaying and network coding, distributed relaying decision and power allocation strategy is applied to obtain the optimal power efficiency. Since only limited CSIs needed in this scheme, it is practical for real applications in two-way relaying networks.

Of course, the DSON suffers from broadcasting the network-coded data according to the weaker link in the third transmission phase for satisfying the requirements of target SNR and target outage probability. From a power saving point of view, the DSON outperforms the ODPA and the SRM in a two-way relaying communication link.

\section{Appendix}

For purposes of completeness, we briefly summarize a result from [16], Lemma A.

Lemma A (see [16]). Random variables $A_{i}, A_{i 1}, \ldots, A_{i K}$, $(i=1, \ldots, M), A_{i 1}, \ldots, A_{i K}$ are independently exponential distributed with parameter $\beta_{i 1}, \ldots, \beta_{i K}$, respectively, $A_{i}=$ $\min \left\{A_{i 1}, \ldots, A_{i K}\right\}$. Let $r=\operatorname{argmax}_{i} A_{i}$, for $F_{A_{r}}(x)$, the $C D F$ of $A_{r}$, there is

$$
F_{A_{r}}(x)=\prod_{i=1}^{M}\left(1-e^{-\beta_{i} x}\right),
$$

where $\beta_{i}=\sum_{k=1}^{K} \beta_{i k}, i=1, \ldots, M$.

\section{Acknowledgments}

This paper was supported by the International Science and Technology Cooperation Program (2008DFA12160), 
China's 863 Project no. 2009AA011501, National Natural Science Foundation of China no. 60832008, China's Major Project no. 2009ZX03003-009, Program for Changjiang Scholars and Innovative Research Team in University (PCSIRT), National Science and Technology Pillar Program no. 2008BAH30B09, and National Basic Research Program of China no. 2007CB310608.

\section{References}

[1] A. Sendonaris, E. Erkip, and B. Aazhang, "Increasing uplink capacity via user cooperation diversity," in Proceedings of the IEEE International Symposium on Information Theory (ISIT '98), p. 156, Cambridge, Mass, USA, August 1998.

[2] P. Larsson, N. Johansson, and K. E. Sunell, "Coded bidirectional relaying," in Proceedings of the IEEE 63rd Vehicular Technology Conference (VTC '06), pp. 851-855, May 2006.

[3] P. Popovski and H. Yomo, "Physical network coding in two-way wireless relay channels," in Proceedings of the IEEE International Conference on Communications (ICC '07), pp. 707-712, Glasgow, Scotland, June 2007.

[4] C.-H. Liu and F. Xue, "Network coding for two-way relaying: rate region, sum rate and opportunistic scheduling," in Proceedings of the IEEE International Conference on Communications (ICC '08), pp. 1044-1049, Beijing, China, April 2008.

[5] R. Ahlswede, N. Cai, S. Y. R. Li, and R. W. Yeung, "Network information flow," IEEE Transactions on Information Theory, vol. 46, no. 4, pp. 1204-1216, 2000.

[6] K. Azarian, H. El Gamal, and P. Schniter, "On the achievable diversity-vs-multiplexing tradeoff in half-duplex cooperative channels," IEEE Transactions on Information Theory, vol. 51, no. 12, pp. 4152-4172, 2005.

[7] J. N. Laneman and G. W. Wornell, "Distributed space-timecoded protocols for exploiting cooperative diversity in wireless networks," IEEE Transactions on Information Theory, vol. 49, no. 10, pp. 2415-2425, 2003.

[8] M. Chen, S. Serbetli, and A. Yener, "Distributed power allocation strategies for parallel relay networks," IEEE Transactions on Wireless Communications, vol. 7, no. 2, Article ID 4450823, pp. 552-561, 2008.

[9] A. Bletsas, H. Shin, and M. Z. Win, "Outage-optimal cooperative communications with regenerative relays," in Proceedings of the 40th Annual Conference on Information Sciences and Systems (CISS '06), pp. 632-637, March 2006.

[10] R. Knopp and P. A. Humblet, "Information capacity and power control in single-cell multiuser communications," in Proceedings of the IEEE International Conference on Communications (ICC '95), vol. 1, pp. 331-335, June 1995.

[11] Q. Ren and Q. Liang, "Opportunistic media access control and rate adaptation for wireless ad hoc networks," in Proceedings of the IEEE International Conference on Communications (ICC '04), vol. 1, pp. 154-158, June 2004.

[12] W. Yang, L. Li, W. Sun, and Y. Wang "Energy-efficient relay selection and optimal relay location in cooperative cellular networks with asymmetric traffic," Journal of China Universities of Posts and Telecommunications, vol. 17, no. 6, pp. 80-88, 2010.

[13] T. E. Hunter and A. Nosratinia, "Distributed protocols for user cooperation in multi-user wireless networks," in Proceedings of the IEEE Global Telecommunications Conference (GLOBECOM '04), pp. 3788-3792, December 2004.
[14] A. Bletsas, A. Khisti, D. P. Reed, and A. Lippman, "A simple cooperative diversity method based on network path selection," IEEE Journal on Selected Areas in Communications, vol. 24, no. 3, pp. 659-672, 2006.

[15] J. N. Laneman, D. N. C. Tse, and G. W. Wornell, "Cooperative diversity in wireless networks: efficient protocols and outage behavior," IEEE Transactions on Information Theory, vol. 50, no. 12, pp. 3062-3080, 2004.

[16] C. Peng, Q. Zhang, M. Zhao, and Y. Yao, "On the performance analysis of network-coded cooperation in wireless networks," in Proceedings of the 26th IEEE International Conference on Computer Communications (INFOCOM '07), pp. 1460-1468, May 2007. 

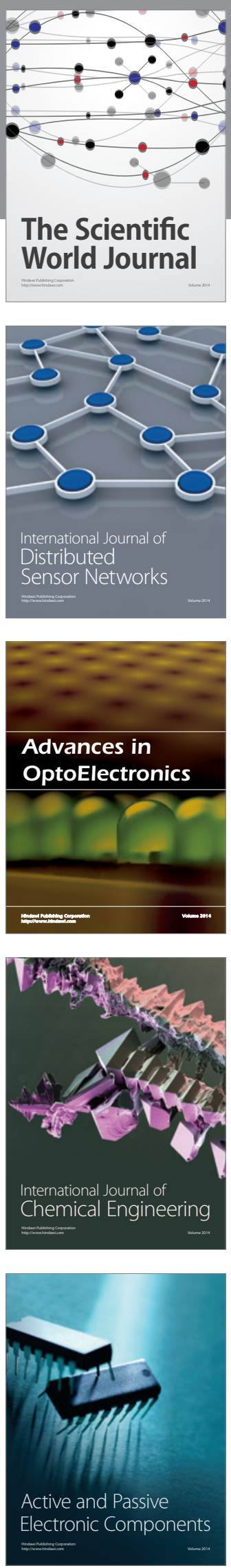
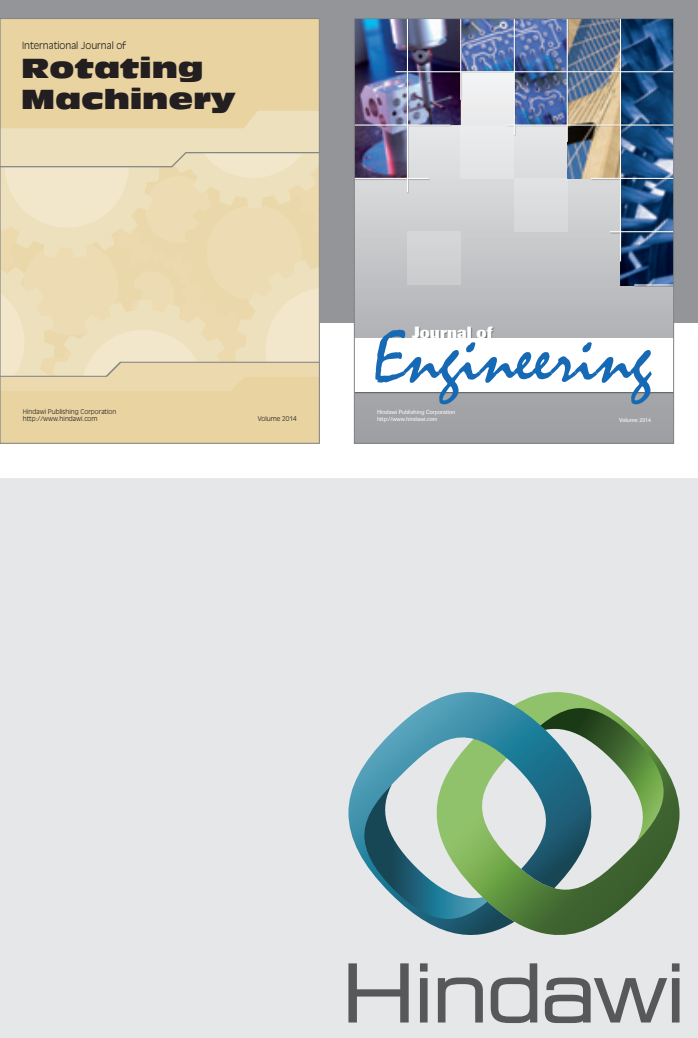

Submit your manuscripts at

http://www.hindawi.com
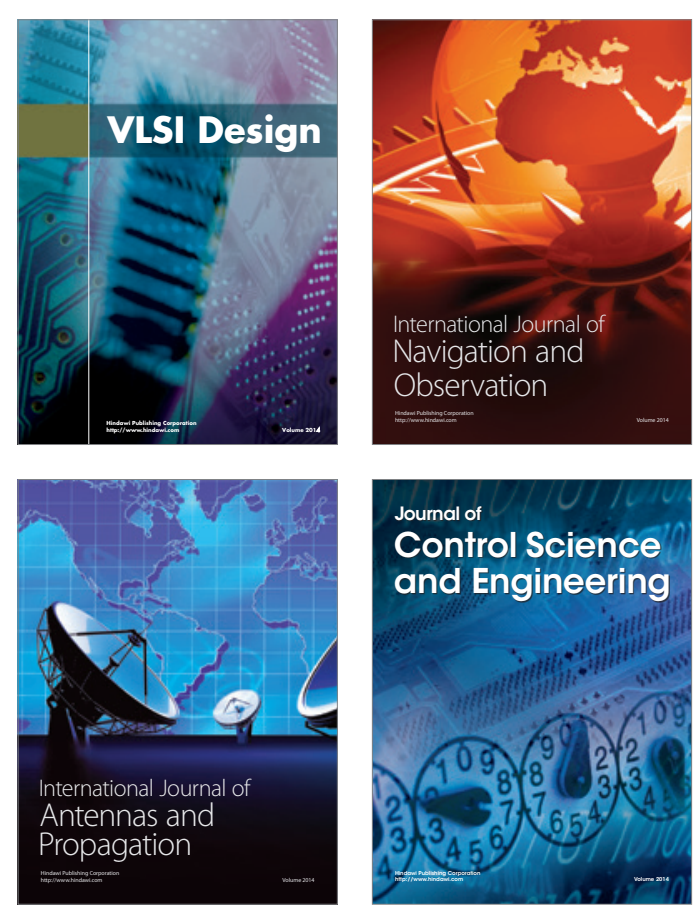
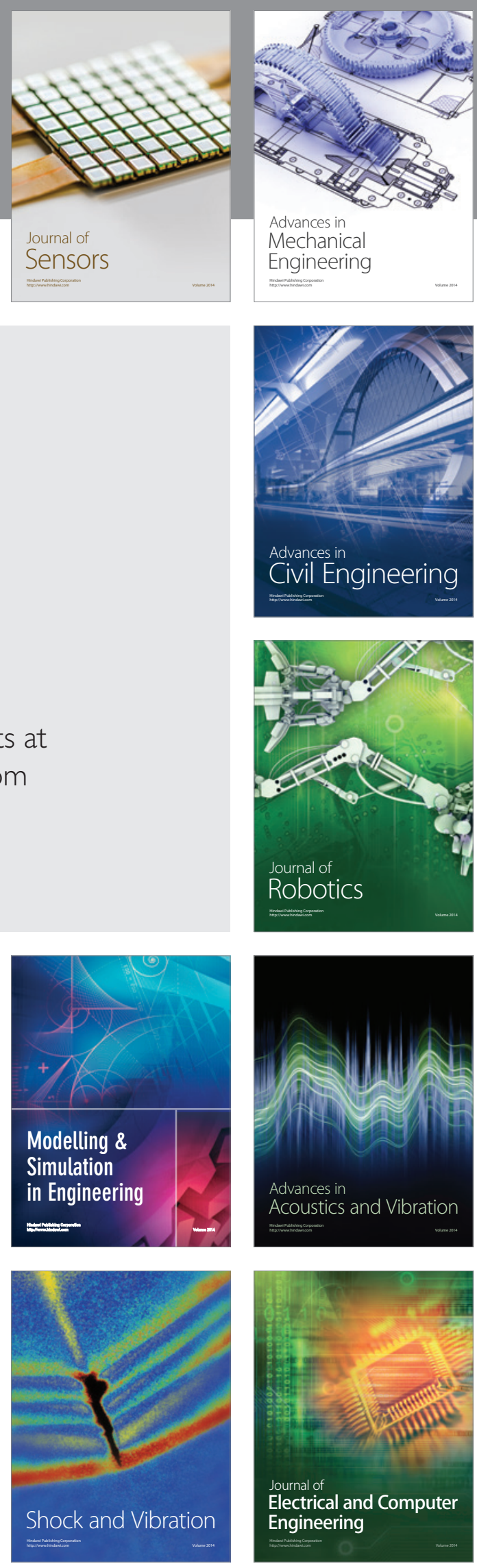